\title{
Development and Use of Cellular Systems to Assess and Correct Splicing Defects
}

\author{
Nuria Suárez-Herrera, Tomasz Z. Tomkiewicz, Alejandro Garanto, \\ and Rob W. J. Collin
}

\begin{abstract}
A significant proportion of mutations underlying genetic disorders affect pre-mRNA splicing, generally causing partial or total skipping of exons, and/or inclusion of pseudoexons. These changes often lead to the formation of aberrant transcripts that can induce nonsense-mediated decay, and a subsequent lack of functional protein. For some genetic disorders, including inherited retinal diseases (IRDs), reproducing splicing dynamics in vitro is a challenge due to the specific environment provided by, e.g. the retinal tissue, cells of which cannot be easily obtained and/or cultured. Here, we describe how to engineer splicing vectors, validate the reliability and reproducibility of alternative cellular systems, assess pre-mRNA splicing defects involved in IRD, and finally correct those by using antisense oligonucleotide-based strategies.
\end{abstract}

Key words $A B C A 4$, Antisense oligonucleotide, Exon skipping, Genetic therapy, Inherited retinal diseases, Maxigene, Midigene, Pre-mRNA, Pseudoexon, Splicing modulation, Splicing vectors

\section{Introduction}

Technologies such as next generation sequencing (NGS) expanded the discovery of genetic variants from coding regions to the entire genome. As a consequence of high-throughput data analysis, it is crucial to be able to correctly identify and distinguish diseasecausing variants from single nucleotide polymorphisms (SNPs). This is especially relevant for intronic variants, as many of them have an unknown functional significance. In the field of inherited retinal diseases (IRDs), a common autosomal recessive condition known as Stargardt disease (STDGl) [1] lacks the bi-allelic molecular diagnosis in $30 \%$ of cases, i.e. the second variant cannot be identified in the coding regions of adenosine triphosphate (ATP) binding cassette type A4 (ABCA4) gene $[2,3]$.

Nuria Suárez-Herrera and Tomasz Z. Tomkiewicz contributed equally to this work. 
According to the Human Gene Mutation Database, mis-splicing mutations have been estimated to represent $8.6 \%$ of the total mutations underlying inherited diseases $(23,868$ / $275,716)$ [4]. In vitro functional assays can provide insight into the underlying mechanisms behind aberrant splicing and identify the mutations that interfere with this process. Currently, the ideal model to assess and correct mis-splicing mutations in STGDI are iPSC-derived retina-like cells (such as photoreceptor precursor cells (PPCs) or retinal organoids) from a patient harboring the genetic variants of interest, as these represent the right cell type(s) with the proper genetic context. In parallel, great efforts have been made to develop a more cost-effective and less time-consuming strategy to reach the same goal, by trying to mimic the pathological situation in a reliable and controlled manner. Engineering and use of multiexon splice vectors have been proven to be extremely effective $[2,5-7]$ in gaining insight into IRDs and, more specifically, STDG1. In general, splice vectors or midigenes refer to a specific type of vector containing a large genomic region that allows to study the splicing processes between the included exons. An even longer genomic content allows for inclusion of long-range cis-acting elements to more accurately reflect the dynamics of splicing [8]. These "artificial" genomic vectors were shown to be very valuable when it comes to $A B C A 4$, as the entire 128 -kb gene has been successfully spanned in a set of midigenes representing an alternative to the impossibility of cloning such a large genomic region in a single vector [6].

The suitability of midigenes for some cell lines reduced the complexity of the study of pathologic deep-intronic variants (among other types of mutations) and their effect on splicing. In addition, validated midigenes harboring splicing variants represent a system for reliable and relatively quick identification of potential therapeutic molecules such as antisense oligonucleotides (AONs). AON-based therapies represent a very effective approach to target mis-splicing mutations. AONs are chemically modified RNA molecules that have the ability of modulating splicing by binding to premRNA and interfering with the spliceosome [9]. They are used in the field of IRDs [10], as well as in other genetic diseases as the purpose of AONs is not limited to splice-switch function only [11]. Generating a reliable artificial splicing system is of major importance when it comes to the development of a potential therapeutic molecule. For the early investigation of possible causative variant affecting pre-mRNA splicing in retinal genes and subsequent assessment of AON potency, midigene technology is a suitable approach that has been shown to produce reliable results $[6,12]$.

Following transfection of the vector into HEK293T cells, AONs are co-transfected, and the splicing correction can be assessed at RNA level. Subsequently, the AONs that are identified 
as most potent can be tested in more advanced and precise cell models such as iPSC-derived retina-like cells.

Despite the proven efficiency of the HEK293T cells, they are not derived from ocular tissue, which allows to speculate whether the splicing dynamics enforced by HEK293T represents that of the retina. As an alternative, with the aim to better represent the retinalike splicing dynamics in in vitro splice assays, we also describe the use of retinoblastoma WERI-Rb-1 cells. These cells are also suitable for midigene transfection and in some cases demonstrate splicing dynamics that are more similar to that of the retina when compared to HEK293T cells.

In this chapter, we describe how to design multi-exon splice vectors followed by appropriate validation and correction of mis-splicing mutations by performing in vitro studies in cellular systems.

\section{Materials}

\subsection{Design of Midigene and Maxigene Splice Vector}

1. Donor and destination vector (see Note $\mathbf{1})$.

2. BP-Clonase cloning kit: BP-Clonase, buffer, proteinase $\mathrm{K}$, etc. (i.e. Gateway ${ }^{\circledR}$ enzymes).

3. LR-Clonase cloning kit: LR-Clonase, buffer, proteinase K, etc. (i.e. Gateway ${ }^{\circledR}$ enzymes).

4. Genomic DNA, in this example, the bacterial artificial chromosome (BAC) clone, CH17-325Ol6 (insert g.94,434, 639-94,670,492), containing the entire $A B C A 4$ gene.

5. Generated midigenes (see Subheading 3.1.1).

6. Primers flanking the region of interest with attB sites. In this chapter we use $A B C A 4$ as an example. Forward primer sequence (attB sites underlined): 5'-GGGGACAAGTTTGTACAAAAAAGCAGGCTTC aacactgctggcaattggag- $3^{\prime}$ and reverse primer sequence: 5'-GGGGACCACTTTGTACAA GAAAGCTGGGTG agctactgtgtggagggtg- $3^{\prime}$. Primers are located in intron 6 and intron 11 of $A B C A 4$, and serve as an example [6].

7. In silico cloning software (VectorNTI, SnapGene or Benchling).

8. High-fidelity Taq polymerase PCR kit: High-fidelity Taq polymerase, dNTPs, buffer, $\mathrm{MgCl}_{2}$ and Q-solution or DMSO if applicable.

9. Unique restriction enzymes for sites present in your constructs, in our example are shown as A and B (see Subheading 3.2.1).

10. Commercially available DNA purification and cleanup kit.

11. Shrimp Alkaline Phosphatase. 
12. T4 ligation kit.

13. Competent cells (preferably commercial ones).

14. LB medium: Autoclave $10 \mathrm{~g} \mathrm{NaCl}, 10 \mathrm{~g}$ tryptone, and $5 \mathrm{~g}$ yeast extract in $1 \mathrm{~L}$ of deionized water.

15. LB plates: Autoclave $10 \mathrm{~g} \mathrm{NaCl}, 10 \mathrm{~g}$ tryptone, $5 \mathrm{~g}$ yeast extract, and $30 \mathrm{~g}$ agar in $1 \mathrm{~L}$ of deionized water.

16. Selection antibiotics (usually ampicillin and kanamycin) at $50 \mathrm{mg} / \mathrm{mL}$ (this is the stock $1000 \times$ concentrated).

17. Commercially available Mini/Midiprep kit for plasmid DNA purification.

18. Electrophoresis equipment and agarose gels.

\subsection{Site-Directed} Mutagenesis
1. Generated midigene and maxigene vectors (see Subheadings 3.1.1 and 3.2.2).

2. Primers to introduce the desired mutation, in this chapter we use the c.859-506G $>\mathrm{C}$ mutation in the $A B C A 4$ midigene as an example (the variant is in bold and underlined):

Forward primer 5' $^{\prime}$ - CTGTGATTTGTTGTTGTTGTTG TTGTTGTTTT G AGACGGAGTAT -TGCTCAG- $3^{\prime}$ and reverse primer 5' - GACACTAAACAACAACAACAACAACAA CAA-AACTCTGCCTCATAACGAGTC-3' [3].

3. Primers to amplify the region within selected restriction sites of the midi-/maxigene.

4. High-fidelity Taq polymerase PCR kit: High-fidelity Taq polymerase, dNTPs, buffer, $\mathrm{MgCl}_{2}$ and $\mathrm{Q}$-solution or DMSO if applicable.

5. Standard Taq polymerase kit.

6. pGEM $^{\circledR}$-T Easy Vector System kit.

7. IPTG and X-Gal.

8. EcoRI restriction enzyme.

9. DpnI restriction enzyme.

10. Competent cells (preferably commercial ones).

11. LB medium: Autoclave $10 \mathrm{~g} \mathrm{NaCl}, 10 \mathrm{~g}$ tryptone, and 5 g yeast extract in $1 \mathrm{~L}$ of deionized water.

12. LB plates: Autoclave $10 \mathrm{~g} \mathrm{NaCl}, 10 \mathrm{~g}$ tryptone, $5 \mathrm{~g}$ yeast extract, and $30 \mathrm{~g}$ agar in $1 \mathrm{~L}$ of deionized water.

13. Selection antibiotics (usually Ampicillin and Kanamycin) at $50 \mathrm{mg} / \mathrm{mL}$.

14. Commercially available Mini/Midiprep kit for plasmid DNA purification.

15. Electrophoresis equipment and agarose gels. 
16. Corresponding restriction enzymes $\mathrm{C}$ and $\mathrm{D}$ (see Subheading 3.2.3).

17. Shrimp Alkaline Phosphatase.

18. Commercially available DNA purification and cleanup kit.

19. T4 ligation kit.

\subsection{Culture Conditions and Cell Lines}

\subsection{Midigene and AON Transfection}

1. HEK293T cells $\left(\right.$ ATCC $^{\circledR}$ CRL-3216 ${ }^{\mathrm{TM}}$ ). Culture medium: DMEM 10\% FCS medium (DMEM medium supplemented with $10 \%$ Fetal Calf Serum (FCS), $100 \mathrm{U} / \mathrm{mL}$ of penicillin, $100 \mu \mathrm{g} / \mathrm{mL}$ streptomycin, and 1\% (v/v) $100 \mathrm{mM}$ sodium pyruvate).

2. WERI-Rb-1 cells (ATCC ${ }^{\circledR}$ HTB-169'M $)$. Culture medium: DMEM 15\% FCS medium (DMEM medium supplemented with $15 \%$ FCS, $100 \mathrm{U} / \mathrm{mL}$ of penicillin, $100 \mu \mathrm{g} / \mathrm{mL}$ streptomycin and $10 \mathrm{~mL}$ of $1 \mathrm{M}$ HEPES).

3. T75 flasks to culture cell lines.

4. $0.25 \%$ trypsin solution for cell dissociation.

5. $1 \times$ PBS.

1. HEK293T or WERI-Rb-1 cells and corresponding culture medium.

2. Midigene vector, as an example we used $A B C A 4$ BA7 midigene [6].

3. AON stock: resuspend the lyophilized AONs at final concentration of $0.1-1 \mathrm{mM}$ in $1 \times$ PBS previously autoclaved twice.

4. 6-well plates and 24-well plates.

5. OptiMEM and transfection reagents (i.e., FuGene ${ }^{\circledR}$ or Lipofectamine $^{\circledR}$ ).

6. $0.25 \%$ trypsin solution for cell dissociation.

7. $1 \times$ PBS.

1. Commercially available RNA isolation kit.

2. Commercially available cDNA synthesis kit.

3. Primers located in the flanking exons of your region of interest. In the example described in this chapter:

(a) Region of interest.

- $A B C A 4$ exon 7 forward: 5'- TCTGAGATCTTGGG GAGGAA-3'.

- ABCA4 exon 8 reverse: 5'-TGGAGTCAATCCCCA GAAAG-3'.

(b) Actin loading control (see Note 2). 
- $A C T B$ exon 3 forward: 5' -ACTGGGACGACATGGA GAAG-3' .

- $A C T B$ exon 4 reverse: 5'-TCTCAGCTGTGGTGGT GAAG-3' .

(c) RHO transfection control.

- $R H O$ exon 5 forward: 5'-ATCTGCTGCGGCAA GAAC- $3^{\prime}$.

- $R H O$ exon 5 reverse: $5^{\prime}$-AGGTGTAGGGGATGGGA GAC- $3^{\prime}$.

4. PCR kit: Polymerase, dNTPs, buffer, $\mathrm{MgCl}_{2}$, water, and Q-solution or DMSO if applicable.

5. Electrophoresis equipment and agarose gels.

\section{Methods}

\subsection{Design of Midigene Splice Vectors}

3.1.1 Gateway Cloning
Retina-specific genes are not readily expressed outside the ocular tissue. The inability to express or poorly express the genes of interest in non-ocular tissues makes it difficult to study variants affecting pre-mRNA splicing. Generation of PPCs from patientderived reprogramed fibroblasts is an alternative to this, but is timeconsuming and expensive.

In here, we describe the generation and use of pCI-NEO-RHO Gateway-adapted in-house vector (see Fig. 1).

1. Identify the gene and sequence of interest in genomic databases such as Ensembl Genome Browser or UCSC [13, 14] and then identify the region of interest. In this case, the region of interest is a deep-intronic variant causing guanine to cytosine substitution at position c.859-506 of the $A B C A 4$ gene. The base substitution strenghtes a cryptic deep-intronic splice acceptor side and causes generation of a 56-nts long pseudoexon between exon 7 and 8 .

2. Design primers suitable for Gateway ${ }^{\circledR} \mathrm{BP}$ cloning ( see Note 3 ).

3. Use the BAC clone, CH17-325Ol6 (insert g.94,434, 639-94,670,492), containing the entire $A B C A 4$ gene. Isolate the BAC DNA using commercially available midiprep kit and use it as a PCR template to generatet the midigenes. Prepare the PCR reaction with $0.5 \mu \mathrm{M}$ of each forward and reverse primer, 0.2 mM dNTPs, Phusion High-Fidelity DNA polymerase, $1 \times$ Phusion GC buffer, 3\% DMSO, and $2.5 \mathrm{ng}$ of BAC DNA in a total of $50 \mu \mathrm{L}$. Run the PCR program where the initial denaturation is at $98{ }^{\circ} \mathrm{C}$ for $30 \mathrm{~s} ; 15-20$ cycles of denaturation at $98{ }^{\circ} \mathrm{C}$ for $10 \mathrm{~s}$ each, annealing at $58{ }^{\circ} \mathrm{C}$ and 


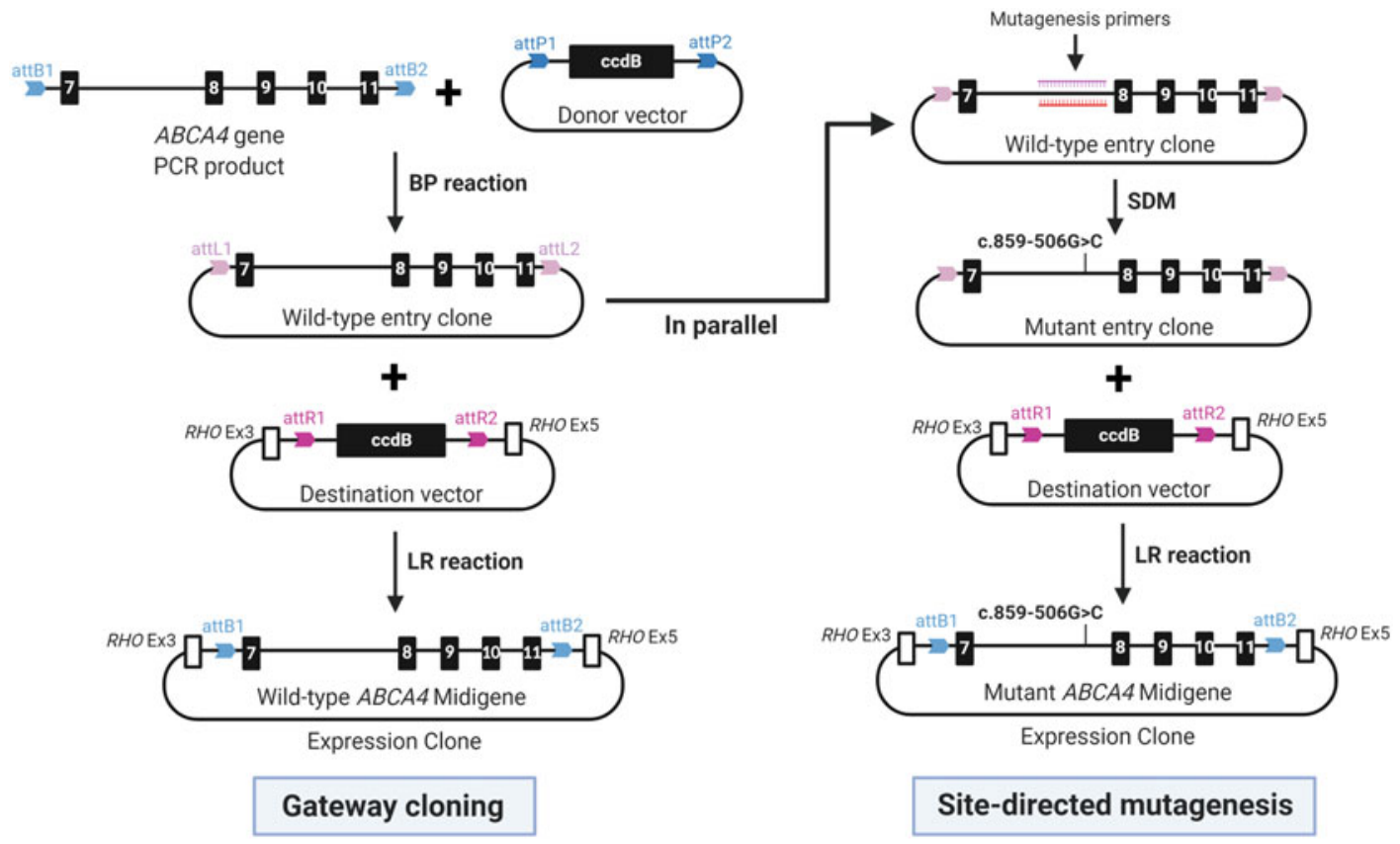

Fig. 1 Schematic representation of wild-type and mutant $A B C A 4$ midigene engineering. The simplified protocol for Gateway ${ }^{\circledR}$ system cloning and site-directed mutagenesis are shown on the left and right section, respectively. SDM: site-directed mutagenesis

extension at $72{ }^{\circ} \mathrm{C}$ for at least 1 min per kb of insert, with a final extension at $72{ }^{\circ} \mathrm{C}$ for $15 \mathrm{~min}[6]$.

4. Resolve the PCR product by gel electrophoresis by loading $10 \%$ of the reaction. The presence of a single band at a corresponding size indicates a succesful PCR amplification step. The band needs to be purified using any available commercial DNA purification and cleanup kit.

5. Set-up the BP reaction as instructed by the manufacturer of the $\mathrm{BP}$-clonase cloning kit. The procedure used in-house includes $1 \mu \mathrm{L}$ of donor vector (150 ng), $2 \mu \mathrm{L}$ of buffer, $150 \mathrm{ng}$ of purified and sequenced PCR product $(\max .5 \mu \mathrm{L})$, milli-Q water up to 8 and $2 \mu \mathrm{L}$ of BP-Clonase enzyme. The BP reaction has to be incubated at $25^{\circ} \mathrm{C}$ for a minimum of $2 \mathrm{~h}$.

6. Terminate the reaction by adding $2 \mu \mathrm{L}$ of Proteinase $\mathrm{K}$ and incubating for $10 \mathrm{~min}$ at $37^{\circ} \mathrm{C}$.

7. Transform up to $5 \mu \mathrm{L}$ of the reaction using competent cells (see Note 4 ) and incubate for $30 \mathrm{~min}$ on ice.

8. Perform the heat shock between 45 and $60 \mathrm{~s}$ at $42{ }^{\circ} \mathrm{C}$ and immediately place the cells back on ice for a minimum of $2 \mathrm{~min}$.

9. Add $250 \mu \mathrm{L}$ of SOC medium into the tube and incubate for $1 \mathrm{~h}$ at $37^{\circ} \mathrm{C}$. 
3.1.2 Side-Directed Mutagenesis
10. Plate the content of the tube on a plate containing the corresponding antibiotic (the in-house vector has a kanamycin-resistance cassette) and incubate $\mathrm{O} / \mathrm{N}$ at $37^{\circ} \mathrm{C}$.

11. Pick between 5 and 10 colonies and grow them in a $3 \mathrm{~mL}$ of LB medium supplemented with the corresponding antibiotic (1: 1000 ratio of antibiotic to medium) $\mathrm{O} / \mathrm{N}$.

12. Perform the plasmid isolation using commercially available miniprep kit for plasmid DNA purification.

13. Verify the presence of insert by conducting restriction analysis. Use donor vector as a control (see Note 5).

14. Sequence the entire wild-type clone to make sure that the Taq did not introduced new mutations during the amplification step (see Note 6).

15. Perform side-directed mutagenesis (see Subheading 3.1.2).

In this section, we describe the side-directed mutagenesis protocol for midigene constructs:

1. Design the mutagenesis primers to have approximatelly 20-nts flanking both regions of the c.859-506 position (see Fig. 1).

2. Prepare mutagenesis mastermix with $1 \mathrm{U}$ of high-fidelity Taq polymerase, $0.5 \mu \mathrm{M}$ of forward and reverse primer, $0.2 \mathrm{mM}$ dNTPs, $1 \times$ high-fidelity reaction buffer, $2 \times$ Q-solution, and 10-35 ng of the wild-type midigene vector in a total of $50 \mu \mathrm{L}$. Run the PCR program where the initial denaturation is at $94{ }^{\circ} \mathrm{C}$ for $5 \mathrm{~min} ; 15$ cycles of denaturation at $94{ }^{\circ} \mathrm{C}$ for $30 \mathrm{~s}$ each, annealing between 50 and $58{ }^{\circ} \mathrm{C}$ for $30 \mathrm{~s}$ and extension time at least $1 \mathrm{~min}$ per each $\mathrm{kb}$ of the complete plasmid with a final extension at $72{ }^{\circ} \mathrm{C}$ for $20 \mathrm{~min}$.

3. Add $1 \mu \mathrm{L}$ DpnI directly to $20 \mu \mathrm{L}$ of the PCR reaction to digest the original template (the wild-type sequence). Incubate the reaction at $37^{\circ} \mathrm{C}$ for $3 \mathrm{~h}$. The reaction is terminated at $80{ }^{\circ} \mathrm{C}$ for $20 \mathrm{~min}$.

4. Perform the transformation using up to $5 \mu \mathrm{L}$ of the reaction (see steps 7-13 in Subheading 3.1.1).

5. Sequence the entire mutant clone to confirm the presense of the desired mutation and also to identify all of the undesired substitutions present in the mutant clone (see Note 6).

There are some variants that may need larger genomic environment in order to correctly assess them. This could be the case for mutations whose effect is only detected when other splice regulatory motifs are present, generally located in introns. As a consequence, larger genomic context should be included and based on our experience, it is difficult to obtain a splicing vector of this size because of several reasons. The first one is the probability of 
inducing single nucleotide changes during the amplification of the genomic region of interest. Long-range PCR and high-fidelity DNA Taq polymerases may prevent this from happening, although these chances increase when amplifying $>10 \mathrm{~kb}$ fragments. Recombination efficiency between the fragment and the donor vector is also affected, as well as the efficiency of site-directed mutagenesis on the entry clone. Both cases are highly associated with the size of the vector. To overcome these limitations, we propose to use the already engineered midigenes completely covering the whole gene in order to generate a maxigene, which combines the genomic context of more than one midigene. In this section, we show an example of the mentioned cloning strategy.

3.2.1 In Silico Design of Maxigene Strategy

3.2.2 Cloning of Maxigene Vectors
1. Select the two midigenes that include the introns and exons of interest.

2. Find a common region within both vectors (generally, it is the overlapping sequence between the first midigene and the following).

3. Look for restriction sites in the common region (A) as well as in the backbone of the vector (B) (see Fig. 2). Restriction sites A and $\mathrm{B}$ should be unique in both midigenes ( see Note 7).

4. Simulate the engineering of the final construct by selecting the fragments flanked by restriction sites $\mathrm{A}$ and $\mathrm{B}$ in both midigenes. From the first vector (based on sequence), select the fragment $\mathrm{B} \rightarrow \mathrm{A}$ (backbone to common region), whereas in the second vector the fragment goes $\mathrm{A} \rightarrow \mathrm{B}$ (common region to backbone).

1. Check if the enzymes are compatible in terms of incubation time/temperature, reaction buffer and stability in order to avoid partial digestions ( see Note 8).

2. Set the digestion reaction for midigene 1 and 2 with restriction enzymes A and B (see Fig. 2). Digest at least $0,5-1 \mu \mathrm{g}$ of DNA and check the digestion product by gel electrophoresis (see Note 9).

3. Cut the band corresponding to the fragment of interest and purify the DNA by using the kit of your convenience (see Note 10).

4. Measure DNA concentration and perform the ligation reaction as described by the manufacturer of the T4 ligase kit (make sure to include a negative control the vector only).

5 . Transform $5 \mu \mathrm{L}$ of the ligation product using DHIO $\beta$ competent cells ( see Note 4) and incubate for 20-30 min on ice.

6. Perform the heat shock for $30 \mathrm{~s}$ at $42{ }^{\circ} \mathrm{C}$ and cool down the cells for $2 \mathrm{~min}$ on ice. 

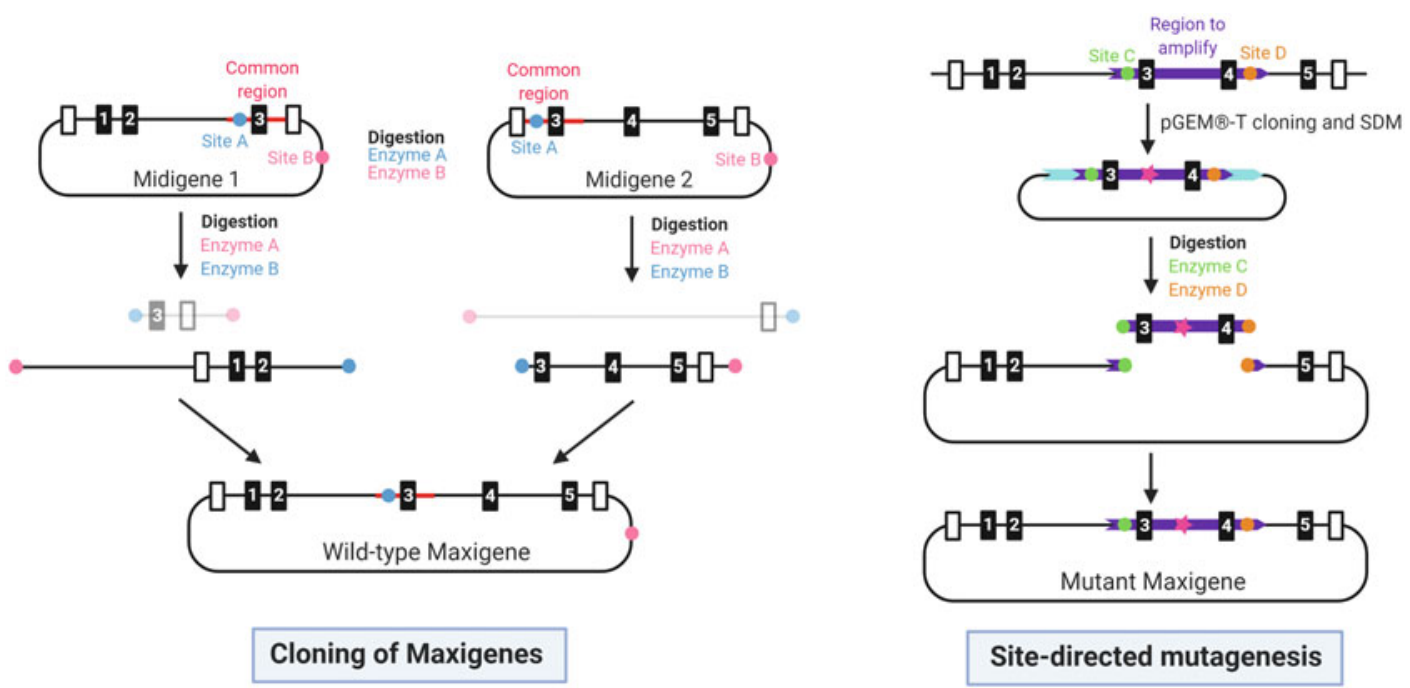

Fig. 2 Schematic representation of wild-type and mutant maxigene engineering. Cloning steps and sitedirected mutagenesis are shown on the left and right section, respectively. This example of maxigene strategy is based on the alternative procedures explained in Subheading 3.2. SDM: site-directed mutagenesis

7. Add $250 \mu \mathrm{L}$ of $10 \beta /$ Stable Outgrowth Medium into the tubes and incubate for $\mathrm{l} \mathrm{h}$ at $37^{\circ} \mathrm{C}$ in the shaking incubator $(250 \mathrm{rpm})$.

8. Plate everything on LB-agar plates containing the corresponding antibiotic (as we are referring to expression clones, these have ampicillin resistance).

9. Incubate $\mathrm{O} / \mathrm{N}$ at $37^{\circ} \mathrm{C}$.

10. Take the plates from the incubator in the morning and in the same afternoon, pick colonies and let them grow at $37{ }^{\circ} \mathrm{C}$ in $3 \mathrm{~mL}$ of LB medium supplemented with the corresponding antibiotic, i.e., ampicillin.

11. Perform plasmid isolation and verify if the ligation worked by restriction analysis or colony PCR. As a negative control, use midigene 1 in parallel.

12. Sequence the positive clones in order to verify that they do not contain any additional mutations.

3.2.3 Site-Directed Mutagenesis
To obtain the mutant maxigene, you can either use midigene 1 or 2 containing the mutation of interest and follow the same protocol indicated in the previous section or perform the site-directed mutagenesis on the wild-type maxigene. In order to perform the second option, we propose the following strategy as an example for a maxigene (see Fig. 2): 
1. Look for unique restriction sites flanking the region of interest (containing the position where single-nucleotide change has to be performed, in this case would be C and D).

2. Amplify this region by using high-fidelity PCR kit.

3. Incubate with normal Taq Polymerase for $20 \mathrm{~min}$ at $72{ }^{\circ} \mathrm{C}$ to add the A overhangs to the final PCR product.

4. Clone the fragment into a $\mathrm{pGEM}^{\circledR}-\mathrm{T}$ vector following the instructions of the kit's manufacturer. Incubate $\mathrm{O} / \mathrm{N}$ at $4{ }^{\circ} \mathrm{C}$.

5. The next day, transform $5 \mu \mathrm{L}$ of the previous reaction using DH5 $\alpha$ competent cells.

6. Perform the heat shock for $60 \mathrm{~s}$ at $42{ }^{\circ} \mathrm{C}$ and cool down the cells for $2 \mathrm{~min}$ on ice.

7. Add $250 \mu \mathrm{L}$ of stable outgrowth medium into the tubes and incubate for $1 \mathrm{~h}$ at $37^{\circ} \mathrm{C}$ in the shaking incubator $(250 \mathrm{rpm})$.

8. Just before plating, add IPTG and X-Gal in the tube and immediately, plate everything on LB-agar plates containing the corresponding antibiotic $\left(\mathrm{pGEM}^{\circledR}\right.$-T vectors have ampicillin resistance).

9. Incubate $\mathrm{O} / \mathrm{N}$ at $37^{\circ} \mathrm{C}$.

10. Pick white colonies and let them grow at $37^{\circ} \mathrm{C}$ in $3 \mathrm{~mL}$ of LB medium supplemented with antibiotic.

11. Perform plasmid isolation using available commercial miniprep kit for plasmid DNA purification, followed by restriction analysis using EcoRI to check if the region is cloned. Keep in mind that your insert may contain an EcoRI restriction site.

12. Sequence the positive clones to check if they contain any additional mutations.

13. Perform site-directed mutagenesis as previously indicated for midigene vectors.

14. Verify the mutation by Sanger sequencing.

15. Digest both the $\mathrm{pGEM}^{\circledR}-\mathrm{T}$ vector and the maxigene with restriction enzymes $\mathrm{C}$ and $\mathrm{D}$.

16. Set the ligation reaction as indicated in the maxigene cloning with either the purified digestion products directly (incubating one of the fragments with phosphatase) or the purified fragments from the agarose gel.

17. Pick colonies and perform restriction analysis or colony PCR to check the correct ligation between the fragment containing the mutation and the rest of the maxigene.

18. Analyze the new inserted region by Sanger sequencing. 


\subsection{In Vitro Evaluation of Splice Vectors in Cell Lines}

\subsubsection{Transfection in} HEK293T
3.3.2 Transfection in WERI-Rb-1
The constructed midi/maxigenes need to be validated and assessed in vitro. In the functional studies of IRDs, the cell line of choice is usually HEK293T. These cells are easy to transfect and do not express retina-specific genes. Therefore, the expression of retinaspecific gene delivered with the vector is not interfered by the endogenous expression of such gene. This advantage is counterbalanced by fact that the HEK293T cells have different properties compared to retina cells. As a consequence, the splicing dynamics are often but not always the same. As an alternative to better represent the retina-like splicing dynamics, we also describe the use of WERI-Rb-1 cells, which are retinoblastoma cells. These cells are still suitable for midigene transfection and sometimes mimic retina-specific splicing patterns better in comparison with HEK293T cells (see Fig. 3).

1. Seed $0.5 \times 10^{6}$ cells/well in DMEM $10 \%$ FCS medium in 6 -well plate if you can transfect the midigene $4 \mathrm{~h}$ post-seeding (see Note 11).

2. Once the cells are attached, transfect $1.2 \mu \mathrm{g}$ of plasmid in a 6-well plate. In this example we used FuGENE (ratio is 3:1 FuGENE:plasmid). To make transfection mix, to $200 \mu \mathrm{L}$ of OptiMEM medium, add 3.6 $\mu \mathrm{L}$ of FuGENE. To the transfection mix add $1.2 \mu \mathrm{g}$ of plasmid and incubate at RT for 15-20 min.

3. Dispense the transfection mix on top of the corresponding well (see Note 12).

4. Incubate the plate at $37{ }^{\circ} \mathrm{C}$ for 24 (see Subheading 3.4.1) or 48 h (see Note 13).

5. After the incubation, gently rinse the cells with pre-warmed $1 \times$ PBS and then detach the cells using $500 \mu \mathrm{L}$ trypsin. Collect the detached cells into a $1.5 \mathrm{~mL}$ Eppendorf using P1000 pipette.

6. Centrifuge the Eppendorf tubes for $5 \mathrm{~min}$ at $1000 \times g$ to pellet the cells, remove the supernatant and wash the cells again in $1 \times$ PBS for $5 \mathrm{~min}$ at $1000 \times \mathrm{g}$.

7. Discard the supernatant and:

(a) freeze the cell pellets at $-80^{\circ} \mathrm{C}$ (storage) after $48 \mathrm{~h}$ incubation and proceed with further analysis at another moment or,

(b) proceed with the RNA isolation (validation of the midigene expression and splicing) after $48 \mathrm{~h}$ incubation.

1. Dispense 1 to $2 \times 10^{6}$ cells to $1.5 \mathrm{~mL}$ Eppendorf tube in a total volume of $500 \mu \mathrm{L}$ of DMEM 15\% FCS.

2. Transfect $1.2 \mu \mathrm{g}$ of plasmid. In this example, we used FuGENE (ratio is 3:1 FuGENE:plasmid). To make transfection mix 


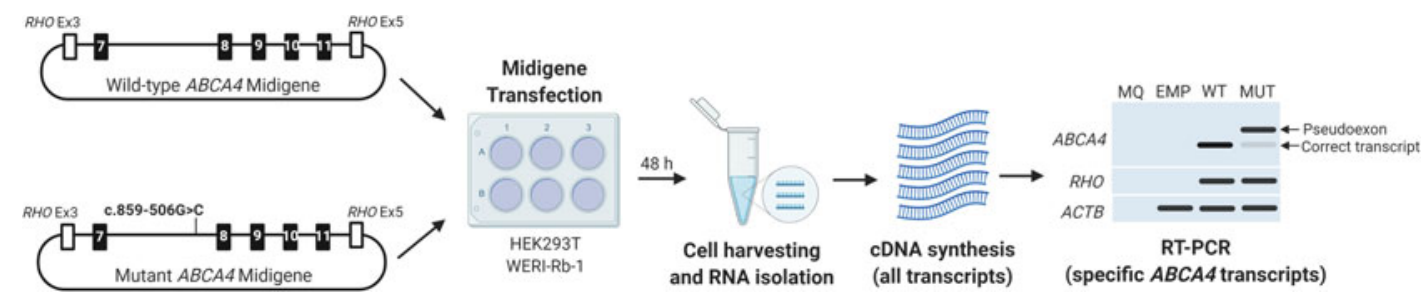

Fig. 3 Experimental strategy to assess splicing defects in HEK293T or WERI-Rb-1 cell lines. The cells are first transfected with a wild-type (WT) or mutant (MUT) ABCA4 midigene and, following a 48-h incubation, splicing is validated by RT-PCR as outlined in Subheading 3.4.3 The gel picture shows an approximate read-out of the expected $A B C A 4$ transcripts. $M Q$ negative control of the PCR reaction, EMP empty transfection mix (endogenous expression of the selected genes within the cell line used)

$200 \mu \mathrm{L}$ of OptiMEM medium with $3.6 \mu \mathrm{L}$ of FuGENE. And to this tube, add $1.2 \mu \mathrm{g}$ of plasmid and incubate at RT for $15-20 \mathrm{~min}$.

3. Dispense the transfection mix to $1.5 \mathrm{~mL}$ Eppendorf tube with WERI-Rb- 1 cells and incubate for $2 \mathrm{~h}$ at $37^{\circ} \mathrm{C}$.

4. After the incubation, transfer the cells to the corresponding well on the 6-well plate and add medium to a total volume of $2 \mathrm{~mL}$.

5. Incubate the plate at $37^{\circ} \mathrm{C}$ for either 24 (see Subheading 3.4.1) or 48 h ( see Note 13).

6. After incubation, collect the cells in $1.5 \mathrm{~mL}$ Eppendorf tube and spin it for $5 \mathrm{~min}$ at $1000 \times g$.

7. Remove the medium and wash the cells again in $1 \times$ PBS for $5 \mathrm{~min}$ at $1000 \times \mathfrak{g}$.

8. Discard the PBS and:

(a) freeze the cell pellets at $-80{ }^{\circ} \mathrm{C}$ (storage) after $48 \mathrm{~h}$ incubation and proceed with further analysis at another moment or,

(b) proceed immediately with the RNA isolation (validation of the midigene expression and splicing) after $48 \mathrm{~h}$ incubation.

3.3.3 Validation of Splicing Events by Reverse Transcriptase PCR (RT$P C R)$
Before starting to use midigenes as an artificial system, it is necessary to check the effect of the variant at pre-mRNA level, and therefore, the RT-PCR is used as a validation method for splicing events.

1. Design forward and reverse primers to flank the region of interest. If the cell line used presents endogenous expression of your target gene, design one of the primers in the artificial exon of the midigene ( $R H O)$ (see Note 14). 


\subsection{Correcting Splicing Defects in Artificial Systems}

3.4.1 Midigene Vector and AON Co-transfection
2. Isolate the RNA from the pellets obtained in Subeading 3.3.1 or 3.3.2 and measure the concentration using NanoDrop.

3. Use $1 \mu \mathrm{g}$ of RNA to synthesize cDNA by following the instruction of the kit's manufacturer.

4. Prepare RT-PCR master mix with 1 U Taq polymerase, $1 \times$ PCR buffer with $\mathrm{MgCl}_{2}, 0.2 \mathrm{mM}$ dNTPs, and 50-60 ng of cDNA in a total volume of $25 \mu \mathrm{L}$. In parallel, prepare the corresponding PCR mixes for actin (loading control) and RHO (midigene transfection control) using the mastermix recipe outlined above with appropriate actin and $\mathrm{RHO}$ primers. Run the PCR program where the initial denaturation is at $94^{\circ} \mathrm{C}$ for $5 \mathrm{~min}$; 35 cycles of denaturation at $94^{\circ} \mathrm{C}$ for $30 \mathrm{~s}$ each, annealing at $58{ }^{\circ} \mathrm{C}$ for $30 \mathrm{~s}$ and extension time at $72{ }^{\circ} \mathrm{C}$ for $3 \mathrm{~min}$, with a final elongation step at $72{ }^{\circ} \mathrm{C}$ for $5 \mathrm{~min}$.

5. Resolve PCR products by gel electrophoresis. Load $10 \mu \mathrm{L}$ from $A B C A 4$ PCR products and $5 \mu \mathrm{L}$ from actin and $R H O$ PCR products as well.

6. Excise the bands coming from each midigene transfection and extract the DNA using a PCR cleanup kit of choice.

7. Measure DNA concentration and if it is not sufficient for Sanger sequencing (minimum of $200 \mathrm{ng}$ per sample), clone the bands in $\mathrm{pGEM}^{\circledR}$-T vector following the manufacturer's instructions.

8. Verify all bands corresponding to different splicing events comparing the mutant to the wild-type condition.

9. Correctly identify and remove midigene artifacts (see Note 15) or heteroduplexes (see Note 16).

In this part of the chapter, we describe the experimental design to correctly compare a set of AON sequences by using midigenes as a model system and how to determine the efficacy of aberrant splicing correction. The utilization of photoreceptor precursor cells and/or retinal organoids is a recommended step in the final stages of $A O N$ potency validation in in vitro studies.

Seeding $0.5 \times 10^{6}$ cells $/$ well in 6 -well plate provides enough cells to seed 6 wells on the 24 -well plate. It is recommended to perform early calculations into how many wells will be required for the splice correction assay as this will influence the number of wells on the 6 -well plate required for midigene transfection. If 6 or less wells on the 24-well plate are needed, one 6-well plate seeded with $0.5 \times 10^{6}$ HEK293T cells and transfected with midigene is enough.

When setting up splice assay with AONs, do not forget about the minimum required controls. These include non-transfected control or empty transfection mix (EMP), cells transfected with 


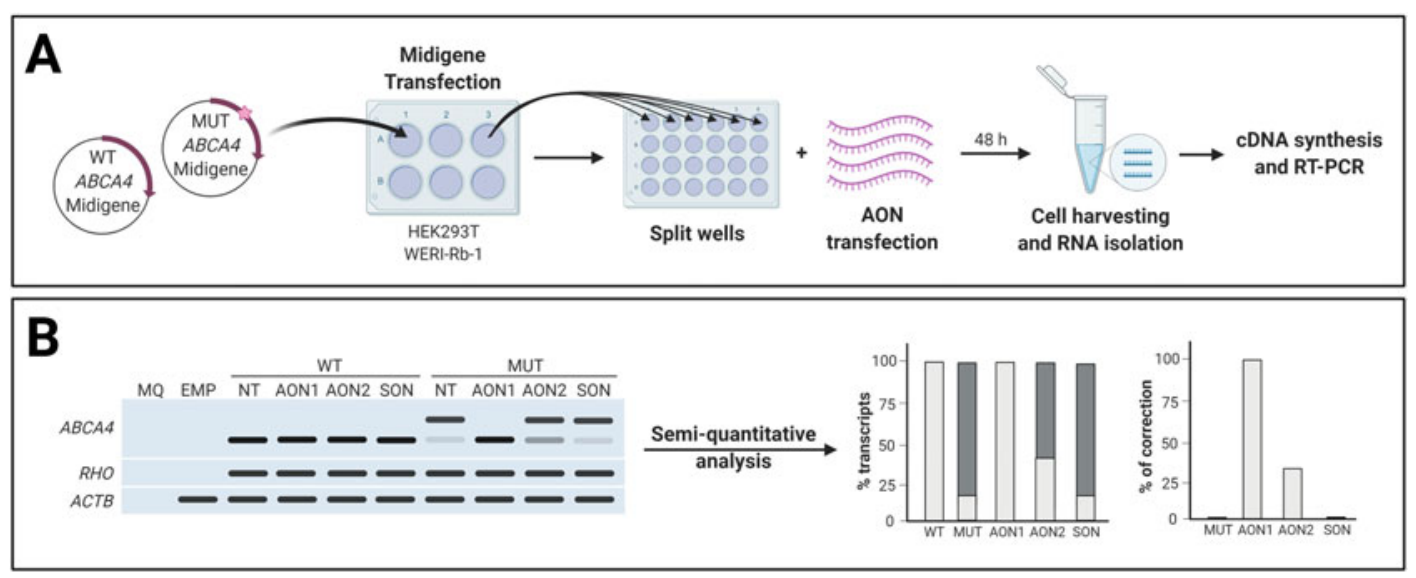

Fig. 4 Analysis of AON-mediated splicing correction in midigene-based splice assays. (a) Experimental strategy to correct splicing defects by co-transfection of wild-type (WT) or mutant (MUT) ABCA4 midigenes with AONs in cell lines as described in Subheading 3.4.1. (b) Representation of splicing read-out on agarose gel and further semi-quantitative analysis. The gel picture represents an approximation of expected $A B C A 4$ transcripts, further semi-quantitative analysis and graphs are based on this estimation. $M Q$ : negative control of the PCR reaction, EMP: empty transfection mix (endogenous expression of the selected genes within the cell line used), NT: non-treated (transfected with midigene but no AONs), SON: scrambled or sense oligonucleotide

wild-type (WT) midigene or mutant (MUT) midigene but not transfected with AONs (NT), and cells transfected with wild-type or mutant midigene and co-transfected with the corresponding scrambled or sense oligonucleotide (SON). The controls cells have to be seeded together with test cells and exposed to the exact same treatment conditions, which include medium change and transfer from 6 -well plate to 24 -well plate.

1. Refer to Subheadings 3.4.1 and 3.4.2 for cell seeding and midigene transfection procedures, then incubate for the next $24 \mathrm{~h}$.

2. Use $500 \mu \mathrm{L}$ of trypsin to detach the HEK293T. Neutralize the effect of trypsin with $500 \mu \mathrm{L}$ of HEK293T specific medium and collect the cells in a $15 \mathrm{~mL}$ Falcon tube. Wash the well with extra $1 \mathrm{~mL}$ of medium to collect all the remaining cells. Add $1 \mathrm{~mL}$ of HEK293T medium to make $3 \mathrm{~mL}$ cell suspension and seed $500 \mu \mathrm{L}$ of the suspension per well in the 24 -well plate using P1000 pipette (see Note 17).

3. Place the 24 -well plate in the $37^{\circ} \mathrm{C}$ incubator and allow for at least $4 \mathrm{~h}$ incubation or until cells become attached to the wells.

4. In this example, the final concentration of the AON was at $0.5 \mu \mathrm{M}$ (see Note 18). To co-transfect two wells on a 24 -well plate (wild-type and mutant midigene) with the same AON at $0.5 \mu \mathrm{M}$, mix $50 \mu \mathrm{L}$ of OptiMEM medium with $1 \mu \mathrm{L}$ of FuGENE $^{\circledR}$. To this mixture add $5 \mu \mathrm{L}$ of resuspended AON at 
3.4.2 Quantification of Splicing Redirection with Image $J$
$100 \mu \mathrm{M}$ stock concentration. Incubate the transfection reaction for $15-20 \mathrm{~min}$ at RT. After incubation add enough medium to make $1 \mathrm{~mL}$.

5. On a 24-well plate, remove all the medium from the transfected wells and gently dispense $500 \mu \mathrm{L}$ of the transfection mix to the wells transfected with wild-type and mutant midigenes. (see Note 19).

6. Place the well plate back in the incubator and allow for $48 \mathrm{~h}$ incubation (see Fig. 4a).

7. After incubation, collect the cells in an $1.5 \mathrm{~mL}$ Eppendorf tube and centrifuge for $5 \mathrm{~min}$ at $1000 \times g$. Remove the medium and wash the cells again in $1 \times$ PBS for 5 min at $1000 \times g$.

8. Discard the PBS and

(a) freeze the cell pellets at $-80{ }^{\circ} \mathrm{C}$ (storage) and proceed with further analysis at another moment or,

(b) proceed immediately with the RNA isolation and the cDNA synthesis following manufacturer's instructions.

9. After the cDNA synthesis perform RT-PCR as previously described (see Subheading 3.3.3).

10. Resolve PCR products by gel electrophoresis.

11. Similar strategy is used for WERI-Rb-1 transfection with AONs. First, refer to Subheading 3.3.3 for midigene transfection to WERI-Rb-1 cells, and follow the protocol outlined above.

Measuring efficiency of an AON-based strategy represents the last step in order to discard or select a potential therapeutic molecule for further clinical studies. There are several methods available to calculate this efficiency and, in this chapter, we focus on a semiquantification strategy for RT-PCR readouts (see Fig. 4b).

1. Open the gel image (TIFF format) in Image J or Fiji (same software).

2. Use the rectangle tool to make selections of the bands you want to quantify (see Note 20).

3. Select the first lane and press Ctrl +1 .

4. Move the newly generated rectangle to the right and put it on top of the next lane, then press Ctrl +2 .

5. Repeat step $\mathbf{4}$ as many times as samples you have in your gel image.

6. After selecting all bands, press Ctrl +3 and a new window with all intensity peaks will appear. 
7. Using the straight-line tool, draw a line at the bottom of every peak and close it in order to remove the background signal from the band intensity signal.

8. Use the wand tracing tool and click on the closed peak to measure its area. Automatically, a new window will appear showing area value while measuring all the peaks ( see Note 21).

9. Copy all area values into an Excel sheet (replace dots by commas if necessary).

10. Calculate the total area of every condition. Use this value as a reference to calculate the \% of correct transcript or aberrant transcript ( see Note 22) as indicated below:

$$
\% \text { of Transcript }=\frac{\text { Area Transcript }}{\text { Total Area }} \times 100
$$

11. Introduce the values in Graphpad Prism and calculate the average of the other replicates as well (see Note 23).

12. Create a graph of the $\%$ of correct/aberrant transcript for every condition.

13. Calculate the percentage of aberrant transcript decrease by setting the aberrant transcript of non-treated mutant midigene value as your reference, this will represent the $\%$ of correction.

14. Take the average of the decrease in all samples per groups and compare the data using a One-Way ANOVA for statistical analysis.

3.4.3 How to Know If an AON Is Effective?
Once you assess the aberrant transcript rescue of the different AONs that are being tested, it is possible to classify them in several groups depending on their splicing redirection efficiency (see Note 24). In previous studies [15], we classified AONs in the following five groups in order to analyze their properties in a straightforward way (see Fig. 4b): highly effective ( $>75 \%$ correction), effective (between $75 \%$ and $50 \%$ correction), moderately effective (between $50 \%$ and $25 \%$ correction), poorly effective (between $25 \%$ and $0 \%$ correction), and noneffective (when correction is not detected).

However, there are some limitations as this protocol is based on a semi-quantitative strategy, such as the interference of heteroduplexes and midigene artifacts. Even though we presented different ways of overcoming these limitations, there is the possibility of using other quantitative strategies [16]. As an example, Fragment Analyzer, TapeStation or digital droplet PCR can be implemented in order to get more accurate splicing readouts (see Note 25). 


\section{Notes}

1. The donor vector used in this example was pDONR201 vector supplied from Invitrogen. The destination vector used was pCI-NEO- $R H O$ vector, which was adapted to contain genomic region encompassing exons 3 through 5 of $R H O$.

2. In the example, we used in-house primers designed for mouse because of the high sequence conservation between mouse $A c t b$ and human $A C T B$ gene.

3. Design the primers to capture as much of the $A B C A 4$ genomic content for the Gateway ${ }^{\circledR}$-adapted vector cloning. The primers have to be able to insert attB sites for Gateway BP cloning.

4. Transformation efficiency for $>15 \mathrm{~kb}$ constructs is significantly increased when using DHIO $\beta$ cells instead of DH5 $\alpha$.

5. Selection of enzyme(s) used to verify the insert depend on the backbone and the insert. We recommend to use an enzyme that cuts in at least two distinct places across the vector. If such enzyme cannot be found, we recommend to use one enzyme that cuts the insert and one enzyme that cuts the backbone of the vector.

6. If new undesired mutations are present within the insert, assess their potential effect on the splicing, in silico, by using tools designed to study pre-mRNA splicing (http://www.umd.be/ HSF/HSF.shtml).

7. If that is not the case, uniqueness of restriction site $\mathrm{A}$ is not strictly needed for both vectors, but it is not recommended having more than two in one of the midigenes. On the contrary, restriction site B should always be unique in both cases. In our case, restriction site $\mathrm{A}$ was located twice in the midigene l. For that reason, we amplified a part of midigene $\mathrm{l}$ including restriction site $\mathrm{B}$ until the beginning of the common region where the second restriction site A was (reverse primer included half of the restriction site A and phosphate group at $5^{\prime}$ ), then we used this product to proceed with the strategy.

8. If this is not the case, sequential digestion is also possible.

9. In our case, the amplified fragment from midigene 1 had to be digested with restriction enzyme B only as restriction site $A$ was located twice in this plasmid, whereas midigene 2 needed sequential digestion with both enzymes $\mathrm{A}$ and $\mathrm{B}$.

10. Based on our experience, even if agarose gels prepared with TAE buffer are recommended to obtain a better resolution of large fragments, the loss of DNA after purification with TAE-specific kits is very high. As an alternative, you can always do a cleanup directly from the digestion product, but 
incubation with phosphatase is needed at least in one of the digested midigenes and screening of colonies afterwards might be more extensive. In our case, phosphatase incubation was performed in digestion product from midigene 2 to avoid re-ligation with itself.

11. Seed at lower density $\left(0.2-0.25 \times 10^{6}\right.$ cells/well $)$ if you have to wait 24 h until transfection.

12. Dispense the transfection mix drop by drop across the well. After dispensing all the midi-/maxigenes to all the wells, gently swirl the plate to ensure homogenous distribution of the midigene.

13. Twenty-four hours incubation is recommended for $\mathrm{AON}$ co-transfection in transcript rescue experiments, $48 \mathrm{~h}$ incubation is recommended for assessing the expression of the wildtype midi-/maxigene or assessing the effect of mutation on the transcript.

14. Using RHO primers for the RT-PCR might reveal other splicing events induced by the artificial exons. However, you can reduce this artifact by performing nested PCR afterwards, substituting the $R H O$ primer by one binding to the next available exon of your transcript.

15. Reduce the amount of transfected midigene if artifacts are masking other splicing events or change the combination of primers, narrowing the region of interest could reduce the amplification of these artifacts.

16. To remove heteroduplexes from the read out, we suggest set a $3-5$ cycles PCR in the same conditions by adding $1 \mu \mathrm{L}$ from the product obtained in step 5. However, this may affect the reliability of the method as one extra PCR step is added, for that reason it is important to validate the observed final readout [17].

17. If you are working with more than one 6-well plate, pool all the cells transfected with the same midigene together. Calculate how much medium needs to be added. If you are working with less than 6 wells on the 24-well plate collect the cells in the total of $3 \mathrm{~mL}$ as described, seed the required number of wells and discard of the remaining cell suspension if not needed.

18. The AONs can be tested at varying molarities to assess the concentration at which AONs are most efficient. The concentration can vary between 0.1 and $1 \mu \mathrm{M}$.

19. As an alternative, it is also possible to remove from each well the exact volume of the transfection mix to be added, making sure the final concentration of AON does not change. 
20. The rectangle size and position (in $\Upsilon$-axis) will be equal for all lanes and cannot be changed in further steps, so make sure to select a region that can cover all bands from the same lane.

21. Try to follow a consistent order through all the analysis, start measuring the band corresponding to the correct transcript and moving to the one of either pseudoexon inclusion (larger) or exon skipping (smaller) band for every different lane/ condition.

22. In the aberrant transcript band, total/partial pseudoexon inclusion or total/partial exon skipping can be counted as aberrant. If heteroduplexes were also included in the band quantification, half of the value of that band should be counted as correct transcript, whereas the other half should be included in the aberrant transcript.

23. It is recommended to repeat the same experiment at least three times (replicates) in order to perform further statistical analysis and make proper comparisons between all conditions

24. This is especially useful when screening a set of AONs with the aim of covering entire regions at pre-mRNA levels [15].

25. In a same study, you can include more than one splicing analysis and compare between them to obtain a more robust result.

This work was supported by European Union's Horizon 2020Marie Sklodowska-Curie Actions grant no. 813490 (to R.W.J.C.) and Retina UK Foundation grant no. GR596 (to R.W.J.C.), Algemene Nederlandse Vereniging ter Voorkoming van Blindheid, Stichting Blinden-Penning, Landelijke Stichting voor Blinden en Slechtzienden, Stichting Oogfonds Nederland, Stichting Macula Degeneratie Fonds, and Stichting Retina Nederland Fonds (who contributed through UitZicht 2015-31 and 2018-21), together with the Rotterdamse Stichting Blindenbelangen, Stichting Blindenhulp, Stichting tot Verbetering van het Lot der Blinden, Stichting voor Ooglijders, and Stichting Dowilvo (to A.G. and R.W.J. C.). This work was also supported by the Foundation Fighting Blindness USA, grant no. PPA-0517-0717-RAD (to A.G. and R. W.J.C.). The funding organizations had no role in the design or conduct of this research. They provided unrestricted grants. 


\section{References}

1. Tanna P et al (2017) Stargardt disease: clinical features, molecular genetics, animal models and therapeutic options. Br J Ophthalmol $101(1): 25-30$

2. Fadaie $\mathrm{Z}$ et al (2019) Identification of splice defects due to noncanonical splice site or deepintronic variants in ABCA4. Hum Mutat 40(12):2365-2376

3. Sangermano R et al (2019) Deep-intronic ABCA4 variants explain missing heritability in Stargardt disease and allow correction of splice defects by antisense oligonucleotides. Genet Med 21(8):1751-1760

4. Stenson PD et al (2017) The human gene mutation database: towards a comprehensive repository of inherited mutation data for medical research, genetic diagnosis and nextgeneration sequencing studies. Hum Genet 136(6):665-677

5. Bauwens $M$ et al (2019) ABCA4-associated disease as a model for missing heritability in autosomal recessive disorders: novel noncoding splice, cis-regulatory, structural, and recurrent hypomorphic variants. Genet Med 21(8):1761-1771

6. Sangermano R et al (2018) ABCA4 midigenes reveal the full splice spectrum of all reported noncanonical splice site variants in Stargardt disease. Genome Res 28(1):100-110

7. Garanto A et al (2016) In vitro and in vivo rescue of aberrant splicing in CEP290associated LCA by antisense oligonucleotide delivery. Hum Mol Genet 25(12):2552-2563

8. Khan $\mathrm{M}$ et al (2019) Identification and analysis of genes associated with inherited retinal diseases. In: Weber BHF, Langmann T (eds) Retinal degeneration: methods and protocols. Springer, New York, pp 3-27
9. Hammond SM, Wood MJ (2011) Genetic therapies for RNA mis-splicing diseases. Trends Genet 27(5):196-205

10. Collin RW, Garanto A (2017) Applications of antisense oligonucleotides for the treatment of inherited retinal diseases. Curr Opin Ophthalmol 28(3):260-266

11. Sharma VK, Sharma RK, Singh SK (2014) Antisense oligonucleotides: modifications and clinical trials. MedChemComm 5(10):1454-1471

12. Tomkiewicz TZ et al (2021) Antisense oligonucleotide-based rescue of aberrant splicing defects caused by 15 pathogenic variants in ABCA4. Int J Mol Sci 22(9):4621

13. Cunningham $\mathrm{F}$ et al (2018) Ensembl 2019. Nucleic Acids Res 47(D1):D745-D751

14. Kent WJ et al (2002) The human genome browser at UCSC. Genome Res 12(6):996-1006

15. Garanto A et al (2019) Antisense oligonucleotide screening to optimize the rescue of the splicing defect caused by the recurrent deepintronic ABCA4 variant c. $4539+2001 \mathrm{G}>\mathrm{A}$ in Stargardt disease. Genes 10(6):452

16. Hiller $\mathrm{M}$ et al (2018) A multicenter comparison of quantification methods for antisense oligonucleotide-induced DMD exon 51 skipping in Duchenne muscular dystrophy cell cultures. PLoS One 13(10):e0204485

17. Thompson JR, Marcelino LA, Polz MF (2002) Heteroduplexes in mixed-template amplifications: formation, consequence and elimination by 'reconditioning PCR'. Nucleic Acids Res 30(9):2083-2088

Open Access This chapter is licensed under the terms of the Creative Commons Attribution 4.0 International License (http://creativecommons.org/licenses/by/4.0/), which permits use, sharing, adaptation, distribution and reproduction in any medium or format, as long as you give appropriate credit to the original author(s) and the source, provide a link to the Creative Commons license and indicate if changes were made.

The images or other third party material in this chapter are included in the chapter's Creative Commons license, unless indicated otherwise in a credit line to the material. If material is not included in the chapter's Creative Commons license and your intended use is not permitted by statutory regulation or exceeds the permitted use, you will need to obtain permission directly from the copyright holder. 